\title{
Character Data Model and Architecture of Garment Based on Global Operation Model
}

\author{
Huosong Xia \\ Department of Information Management and Information System, Wuhan University of \\ Science \& Engineering, Wuhan 430073, Hubei, P.R. China bxxhs@sina.com \\ Eller College of Management, University of Arizona, Tucson 85721, Arizona, USA, \\ bxxhs@email.arizona.edu
}

\begin{abstract}
With the rapid development of global economy, more and more companies begin to set up global operation model (GOM). However, some applications do not bring forecasting benefits, because business models in GOM are different from the traditional business environments. Garment is the traditional industry. It needs to develop better business models and strategy to adapt to the global economy. What is the most important is to learn bow to use IT to improve operation processing. An aim of garment operation model is to ensure that knowledge is shared and that the right information and knowledge is available in the right forms to the right entities at the right times for the right costs with the maximum integrated organizations. However, the processing of garment operation model is a complex system related to character data model, IT infrastructure and others that links them together with GOM. The GOM will have profound impact on the garment operation model. In this paper, we discuss garment character model based on GOM. Firstly, the key factors of GOM are summarized. Secondly, the character data model is set up, which is related to several definitions. Thirdly, the architecture of garment industry based on GOM is presented and the components of the architecture are analyzed.
\end{abstract}

Keywords: Key factors of global operation model (GOM), Garment industry, Made to measure (MTM), Character data model, Architecture, Knowledge diffusion

\section{INTRODUCTION}

The issue of global operation has emerged rapidly. To achieve a sustainable competitive advantage companies must understand and consider building effective business operation model. Garment industry has become one of the most important considerations to garment industry in today's demand-driven economy as customers are not just buying productive products but personality products. Due to the global economy and the new IT era, the way to obtain competitive advantage is different from that in the traditional era and thus garment industry has to develop better business strategies based on global operation model (GOM). The ultimate goals of garment industry are to acquire new customers, retain old customers and grow customer profitability by building good business model. The most important is to understand and to meet customers' expectations, to reach agreement on performance and on value requirements. With regard to the new environment, we should need a

Please use the following format when citing this chapter:

Xia, H., 2007, in IFIP International Federation for Information Processing, Volume 255, Research and Practical Issues of Enterprise Information Systems I] Volume 2, eds. L. Xu, Tjoa A., Chaudhry S. (Boston: Springer), pp. 1419-1423. 
new perspective for garment industry to adapt garment industry based on GOM. This paper will provide an understanding of and appreciation for the GOM. For such purposes, several aspects are decided as the priorities for the discussion in this paper. Section 2 presents the key factors of GOM. Section 3 presents the character data model of garment EC. Section 4 presents an Architecture based on GOM. And Section 5 offers some conclusions.

\section{KEY FACTORS OF GLOBAL OPERATION MODEL}

There are many global operation factors. The model of the key ones is described as figure 1 .

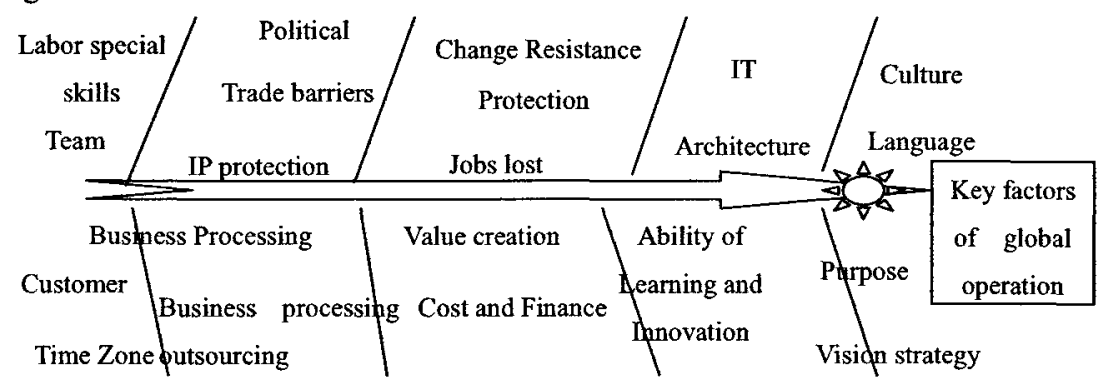

Figure 1. Key Factors of GOM

Why do enterprises adopt the global operation? Both internal and external factors must be taken into consideration. From the internal factors perspectives, the vision strategy including purpose, ability of learning and innovation, value creation, business procession, and customer is considered. From the external factors perspectives, the different national political, change resistance, source of labor special skills, IT architecture, culture and language are considered. To garment enterprise, we need to analyze to detail character data model of garment based on GOM and the architecture.

\section{CHARACTER DATA MODEL OF GARMENT INDUSTRY}

We give the following character data model of garment Industry:

Definition 1, given $F=(M, T, S, P, R, D)$ representing character data description for garment EC,F is also the set of customer garment description (f), $M$ as a set of customers measurement chart in different dimensions, $M=\{m i \mid i=1,2,3 \cdots n\}$, $T$ as a set of garment and customer character's type, $T=\{t\}, S$ as a set of style, $S=\{\mathrm{S}\}, \mathrm{P}$ as a set of paper sample , $P=\{p\}, R$ as a set of altering a design rules, $R=\{r\}, D$ as a set of customer's demand description, $\mathrm{D}=\{\mathrm{d}\}$, the customer order garment at the time, $\mathrm{F}: \mathrm{M}$ $X \mathrm{D} \rightarrow \mathrm{P}$.

Definition 2, $\mathrm{M}=\{\mathrm{mi} \mid \mathrm{i}=1,2,3 \cdots 20\}, \mathrm{m} 0$ representing customer $\mathrm{id}, \mathrm{m} 1$ representing height, $\mathrm{m} 2$ representing neckline, $\mathrm{m} 3$ representing chest line, $\mathrm{m} 3$ representing chest, 
$\mathrm{m} 4$ representing waist, $\mathrm{m} 5$ representing seat, $\mathrm{m} 6$ representing cross-front $(\mathrm{CF}), \mathrm{m} 7$ representing cross-back $(\mathrm{CB}), \mathrm{m} 8$ representing half shoulder width, $\mathrm{m} 9$ representing sleeve length, m10 representing $\mathrm{CF}$ length, $\mathrm{m} 11$ representing $\mathrm{CB}$ length, $\mathrm{m} 12$ representing hanging point to front waist, $\mathrm{m} 13$ representing hanging point to back waist, $\mathrm{m} 14$ representing upper seat rise, $\mathrm{m} 15$ representing seat rise, $\mathrm{m} 16$ representing inseam, $\mathrm{m} 17$ representing crotch length, $\mathrm{m} 18$ representing measure time, $\mathrm{m} 18$ representing male or female.

Definition 3, $\mathrm{T}=\{\mathrm{tij} \mid \mathrm{i}=1,2,3,4,5, \mathrm{j}=1,2,3,4,5,6\}, \mathrm{i}=1$ representing extended big type, $i=2$ representing big type, $i=3$ representing extended middle type, $i=4$ representing middle type, $i=5$ representing small type; $j$ representing typical character customer type. The garment business process for EC based on made to measure (MTM) is described as figure 2.

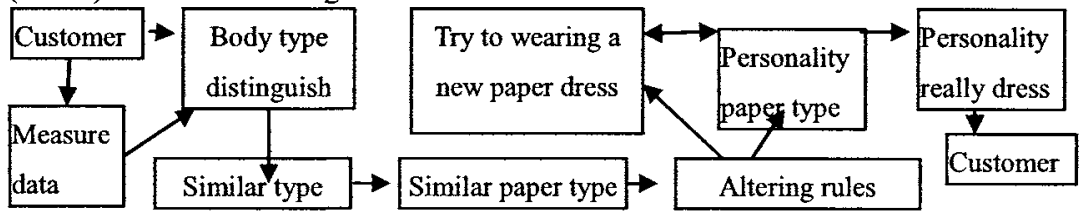

Figure 2. The Garment Business Process Based on MTM

Measure data, body type, paper sample type, rules and other customer characters are stored in DB. To be described by computer, all data must be provided with a metadata structure model definite, unanimous marks and a proper system built.

According to the process of garment business which can be divided into measure data process, distinguish process, altering process, and simulation process; the first category can be coded in DB, which can be easily obtained in three-dimension garment measure system; the rest three process kind are the knowledge of hidden and judgment, whose knowledge can just be sensed but not be explained inwards.

The feature of garment business model is summarized as Table 1. As we know, garment industry has a big impact on industrial standards. Most garment industry models are not quite mature at the current stage, and there are a little leaders garment companies. The integration of business model is imperative based on different standards payment and exchange documents smoothly.

Table 1. Feature of Garment Business Model

\begin{tabular}{|c|c|c|c|l|c|c|}
\hline Feature & $\begin{array}{l}\text { Industrial } \\
\text { standards }\end{array}$ & Mature & Complexity & $\begin{array}{l}\text { Measure } \\
\text { cost }\end{array}$ & Personality & $\begin{array}{l}\text { Sense \& } \\
\text { feeling }\end{array}$ \\
\hline Degree & Less & Less & More & High & High & High \\
\hline
\end{tabular}

\section{AN ARCHITECTURE OF GARMENT INDUSTRY BASED ON GOM}

In fast-cycle garment industries such as fashion garment industry, where the product's life span is relatively short, perhaps one month or less, so time-to market is important. For this purpose, we propose the architecture of garment based on GOM 
and new IT as shown in Figure 3. The components of the architecture of garment based on GOM are described as follows.

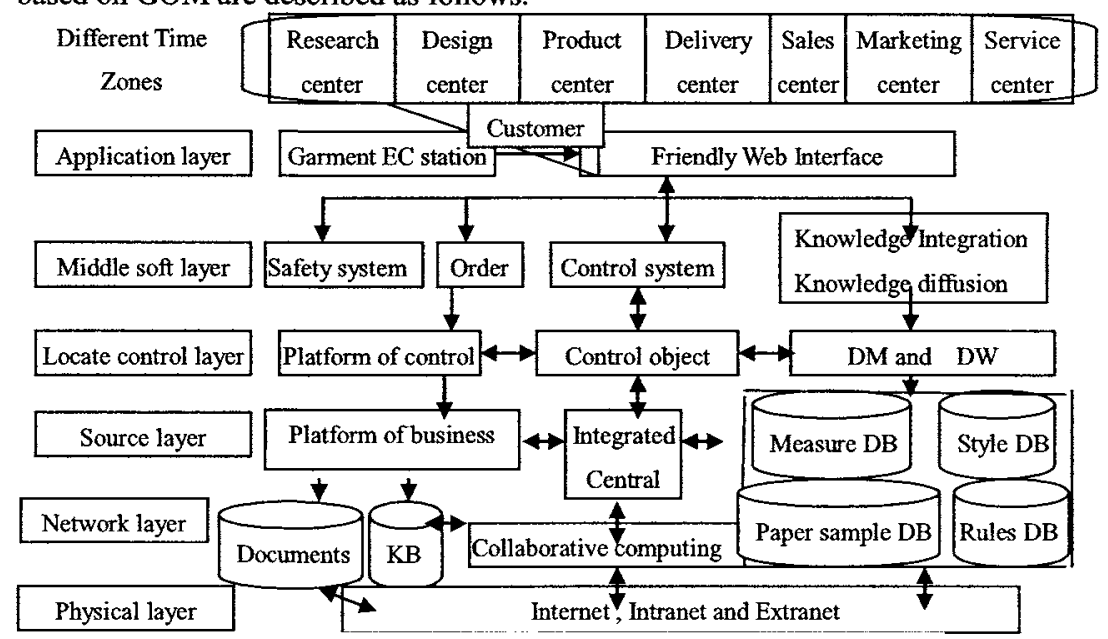

Figure 3. An Architecture of Garment Based on GOM

The users include research center, design center, product center, delivery center, sales center, marketing center, service center, and maybe customers. They are distributed all over the world, possibly in different time zones. The architecture can ensure the right delivery and sharing of the information and knowledge diffusion.

EC station and friendly Web interface are located in application layer of the architecture, which brings integrated capabilities of garment order, knowledge integration and knowledge diffusion, control system, and safety system. The station plays an important role in the coordination of plans for information and knowledge acquisition and knowledge diffusion.

Source layer is located in the fourth layer including source agent and platform of business from integrated central, measure DB, style DB, paper sample DB, rules DB, $\mathrm{DW}, \mathrm{KB}$, and documents as Table 2 .

Table 2. Data Source of Garment EC

\begin{tabular}{|l|l|l|l|}
\hline Type & Description & Type & Description \\
\hline Basic data & Raw data and profile & Paper sample & paper design fore-product \\
\hline Measure & As definition 2 & visit log & $\begin{array}{l}\text { customer' behavior at the EC } \\
\text { site such as the fist visit time } \\
\text { (fvist),last visit time (lvisit) }\end{array}$ \\
\hline Styles & As definition 3 & &
\end{tabular}

Network layer is located in the fifth layer including network management protocol, code management protocol, compute management protocol, storage management protocol, and service protocol. The main aim is collaborative computing.

Locate control layer consists of system capture process designs, histories of process instances, search and retrieval capabilities for information and knowledge. 
Integrated central must meet collaborative processes controlling requirement available to the right processes at the right time and the right place for building and sustaining an organization's competencies.

Centers in different time zones can provide collaborative and virtual environment for automatic product and sales cycle including recommendations match customer interests. High customer satisfaction can lead to increased customer retention and loyalty, resulting in positive economic outcomes. The centralized customer including measure data is clustered by DM and DW that generates visual diagrams showing all clusters with labeled features of clusters. Designers and analysts assign classes to clusters based on the labeled features of clusters. The clusters of data with class labels serve as personality paper type for garment industry and inductively inference the characteristic rules for the customers in normal, defected, possibly defected classes.

\section{CONCLUSIONS}

The key factors of GOM are summarized. Based on the GOM, the character data model and the architecture of garment $\mathrm{EC}$ have been presented by this paper. Through analysis of above, this research architecture and key factors of GOM provide a basis for understanding the competitive predisposition of a firm as it enters into a program. It is, therefore, necessary to develop better, more effective, and more accurate understanding of garment industry based on GOM. Our understanding of the garment industry is still quite limited.

The future work of enhancing the model and architecture is as follow: Use the model and architecture to explore more aspects of application. Build a knowledge integration and knowledge diffusion system for garment industry based on GOM.

\section{ACKNOWLEDGEMENTS}

This research was supported by National Philosophy and Social Science Fund (07BTQ010) of China, HuBei Province Fund of China (2007097, D200517003, 20040216), WUSE Fund (20063102). We are deeply grateful to Qian Yang for her help in editing this paper.

\section{REFERENCES}

1. A. Gupta, S. Seshasai, S. Mukherjo and O. Ridge, Offshoring: The Transition from Economic Drivers toward Strategic Global Partnership and 24-Hour Knowledge Factory, Journal of Electronic Commerce in Organizations. Volume 15, Number 2, pp.1-23, (2007).

2. S. Seshasai, A. Gupta, and A. Kumar, An integrated and collaborative framework for business design: A knowledge engineering approach, Data \& Knowledge Engineering. Volume 52, pp.157-179, (2005). 